\title{
Short communication: Dynamic changes in bacterial diversity during the production of powdered infant formula by PCR-DGGE and high-throughput sequencing
}

\author{
Zhi-Qiang Xiong, ${ }^{1} \oplus$ Ying-Ying Li, ${ }^{1}$ Yu-Wei Xiang, ${ }^{1}$ Yong-Jun Xia, ${ }^{1} \oplus$ Hui Zhang, ${ }^{1}$ Shi-Jie Wang, ${ }^{2,3}$ \\ and Lian-Zhong $\mathrm{Ai}^{1 *}$ (1) \\ ${ }^{1}$ Shanghai Engineering Research Center of Food Microbiology, School of Medical Instrument and Food Engineering, \\ University of Shanghai for Science and Technology, Shanghai 200093, China \\ ${ }^{2}$ College of Bioscience and Bioengineering, Hebei University of Science and Technology, Shijiazhuang 050018, China \\ ${ }^{3}$ Shijiazhuang Junlebao Dairy Co. Ltd., Shijiazhuang 050211, China
}

\section{ABSTRACT}

Microorganisms such as thermophilic and psychrotrophic bacteria cause spoilage of milk and milk products [e.g., powdered infant formula (PIF)], mainly because they produce heat-stable extracellular enzymes. However, the dynamic changes in microbial diversity during PIF production are still not well understood. We used denaturing gradient gel electrophoresis (DGGE) and high-throughput sequencing (HTS) to investigate bacterial community structure and distribution during the major stages of PIF production: raw milk, pasteurization, mixing, evaporation, and spray-drying. Our PCR-DGGE analysis indicated that Lactobacillus and Pseudomonas spp. were the dominant bacteria at the raw milk and pasteurization stages; Lactococcus, Streptococcus, Enterococcus, and Lactobacillus spp. were abundant during mixing, evaporation, and spraydrying. Our HTS analysis showed that Pseudomonas had an abundance of $96.79 \%$ at the raw milk stage. Lactobacillus, Streptococcus, Thermus, Acinetobacter, and Bacteroides spp. were most common after pasteurization. The index of bacterial diversity was highest at the evaporation stage, suggesting a high potential risk of microbial contamination. The results from DGGE and HTS were consistent in reflecting changes in dominant flora, but different in reflecting the richness of bacterial communities present during PIF production: HTS revealed a much higher richness of bacterial species than DGGE. Our findings from DGGE and HTS showed that psychrophilic and thermophilic bacteria were the main flora present during PIF production: psychrophilic bacteria were mainly Pseudomonas spp. and thermophilic bacteria were mainly Lactobacillus,

Received December 16, 2019.

Accepted February 11, 2020.

*Corresponding author: ailianzhong@hotmail.com
Streptococcus, and Bacillus spp. To our knowledge, this is the first study to report dynamic changes in microbial communities during PIF production. Our results provide insight into bacterial communities and identify potential contamination sources that could serve as a guide for reducing microbial risk.

Key words: powdered infant formula, bacterial diversity, denaturing gradient gel electrophoresis (DGGE) analysis, high-throughput sequencing

\section{Short Communication}

As an effective breast milk substitute, powdered infant formula (PIF) is an important source of nutrition for the normal growth and development of infants. Milk is the raw material for PIF, and it is an excellent medium for microbial growth owing to its nutritional composition and water content. During PIF production, contaminating bacteria are normally acquired during milking, handling, and processing. Bacterial counts in fresh milk from healthy cows are $10^{2}$ to $10^{5}$ cfu/mL (Yuan et al., 2012; Delgado et al., 2013); levels can be affected by a range of factors, including season, environmental hygiene, and cooling efficiency (Doyle et al., 2016). Raw milk is generally stored at low temperatures to inhibit microbial growth. Most microorganisms (e.g., psychrotrophic bacteria) that grow in milk are temperature-sensitive, and levels decrease substantially after pasteurization. However, the surviving microorganisms - mainly lactobacilli, micrococci, and sporeforming bacteria (Yuan et al., 2012) - are resistant to high temperatures. Therefore, pasteurized milk comes with a risk of recontamination by surviving microflora. As well, bacteria can develop biofilms on dairy surfaces to protect themselves from adverse environments such as cleaning-in-place processes and pasteurization (Sadiq et al., 2017). Spoilage caused by mesophilic microorganisms can be reduced by controlling processing tem- 
peratures, but this approach cannot prevent spoilage by psychrotrophic microorganisms. Thermophilic and psychrotrophic bacteria cause spoilage during PIF production by secreting heat-stable extracellular enzymes such as lipases and proteases (Hantsis-Zacharov and Halpern, 2007; Ziyaina et al., 2018). These extracellular enzymes are usually thermostable and can maintain their activity even after UHT treatment. Therefore, monitoring microbial contamination is critical for the safety and quality of PIF.

A series of culture-dependent and -independent techniques have been developed to detect microorganisms, including high-throughput sequencing (HTS) and denaturing gradient gel electrophoresis (DGGE). Compared with traditional plate culture methods, HTS can be used obtain a more comprehensive picture of an entire microbial population, including both culturable and unculturable microorganisms (Quigley et al., 2012). Wu et al. (2018) used culture and HTS to investigate microbial communities in the air of a PIF factory. Zheng et al. (2016) also used HTS to analyze the bacterial diversity of 30 Chinese domestic and imported PIF products. However, studies of microbial communities during PIF production are very limited. In this study, we used DGGE and HTS to estimate dynamic changes in bacterial diversity and composition (especially thermophilic and psychrophilic bacteria) during PIF production.

The main stages of PIF production are raw milk, pasteurization, mixing, evaporation, and spray-drying (Supplemental Figure S1; https://doi.org/10.3168/jds .2019-18064). To provide an overview of the dominant bacteria present during PIF production, we undertook PCR-DGGE using universal primers (Supplemental File S1 and Table S1; https://doi.org/10.3168/jds.2019 -18064) to analyze bacterial $16 \mathrm{~S}$ rRNA. We optimized DGGE conditions by selecting the suitable hypervariable regions of $16 \mathrm{~S} \mathrm{rRNA}$ and DGGE gradients (Supplemental Figure S2; https://doi.org/10.3168/jds .2019-18064). The number of bands on each lane of the DGGE represents the species diversity of the sample, and the brightness of the bands indicates the richness of species. The $16 \mathrm{~S}$ rRNA variable region V3 to V5 and DGGE gradient 25 to $70 \%$ had the best separation and most amplified fragments of PIF samples (Supplemental Figure S2). As shown in Figure 1, the DGGE patterns for these 5 main stages were completely different, suggesting that the dominant bacteria changed dynamically during PIF production. The diversity index of DGGE in different stages was calculated using Quantity One software (Bio-Rad, Hercules, CA; Supplemental Table S2; https://doi.org/10.3168/jds .2019-18064). The Shannon-Wiener index indicated that bacteria were most abundant in the spray-drying stage $(2.43 \pm 0.042)$, followed by the evaporation stage $(2.26 \pm 0.059)$, the mixing stage $(2.01 \pm 0.066)$, the raw milk stage $(1.79 \pm 0.067)$, and the pasteurization stage $(1.71 \pm 0.099)$. The pasteurization stage had the lowest diversity index, confirming that pasteurization is an efficient strategy for reducing the levels of microorganisms in milk. We also used an unweighted pair group method with an arithmetic mean clustering diagram to display the genetic relationships between the different stages of PIF production (Supplemental Figure S3; https://doi .org/10.3168/jds.2019-18064). We separated the 5 stages into 2 large branches: evaporation and spray-drying formed one branch; and raw milk, pasteurization, and mixing formed the other. The DGGE profiles suggested that bacterial diversity was similar for the evaporation and spray-drying stages, and for raw milk, pasteurization, and mixing. We identified the numbered bands in Figure 1 by DNA extraction, PCR amplification, and sequencing (Supplemental Table S3; https://doi.org/ 10.3168/jds.2019-18064). The brightest band for the raw milk, pasteurization, and mixing stages (band 5) represented the genus Lactobacillus. The main genera in the raw milk stage were Lactobacillus and Pseudomonas. The main species in the pasteurization stage were Pseudomonas fragi and Bacillus licheniformis, showing that Pseudomonas may be transferred from raw milk to pasteurization. Bacillus licheniformis is one of the dominant thermophilic bacteria in Chinese commercial milk powders (Yuan et al., 2012). Streptococcus thermophilus and Lactococcus lactis were the prominent species in the evaporation and spray-drying stages, and these are also commonly found in other milk products. Callon et al. (2007) found that common bacterial species in milk such as Lactococcus lactis, Lactococcus garvieae, Lactobacillus casei, and Pseudomonas putida were also found in cheeses. Aponte et al. (2008) monitored microbial community during cheesemaking and found that Streptococcus thermophilus, Lactobacillus rhamnosus, and Streptococcus macedonicus were prevalent.

Compared with DGGE, HTS provides higher-level, richer data for bacterial diversity, as well as a greater amount of data. Unfortunately, we failed to construct a cDNA library for the pasteurization stage, so we analyzed bacterial diversity using HTS for only 4 of the 5 stages of PIF production. We obtained a total of 147,534 raw reads from HTS: 31,803 for the raw milk stage, 36,893 for the mixing stage, 39,254 for the evaporation stage, and 39,584 for the spray-drying stage. The paired-end sequences of these reads were overlapped and assembled to obtain high-quality $16 \mathrm{~S}$ rRNA sequences, of which the average length was 447 bp. The data volume of these high-quality sequences was sufficient to determine most bacterial types, because all Shannon rarefaction curves reached saturation 
and had high Good's query coverage (>99\%). According to $97 \%$ species identity, we found 1,720 operational taxonomic units (OTU) of bacterial communities in the 4 stages (Figure 2): $128(7.44 \%)$ in the raw milk stage, $478(14.01 \%)$ in the mixing stage, $537(31.22 \%)$ in the evaporation stage, and $577(33.54 \%)$ in the spraydrying stage (Figure 2), among which 84 (4.88\%) OTU were shared across the 4 stages. Based on the minimum sequence numbers across all stages, we obtained bacterial richness and diversity from randomly selected sequences (Supplemental Figure S4; https://doi.org/ 10.3168/jds.2019-18064). The richness indices Chao1, Sobs (observed species richness), and ACE (abundancebased coverage estimator) showed that the raw milk stage had low bacterial diversity and the spray-drying stage had high diversity. At the mixing, evaporation,

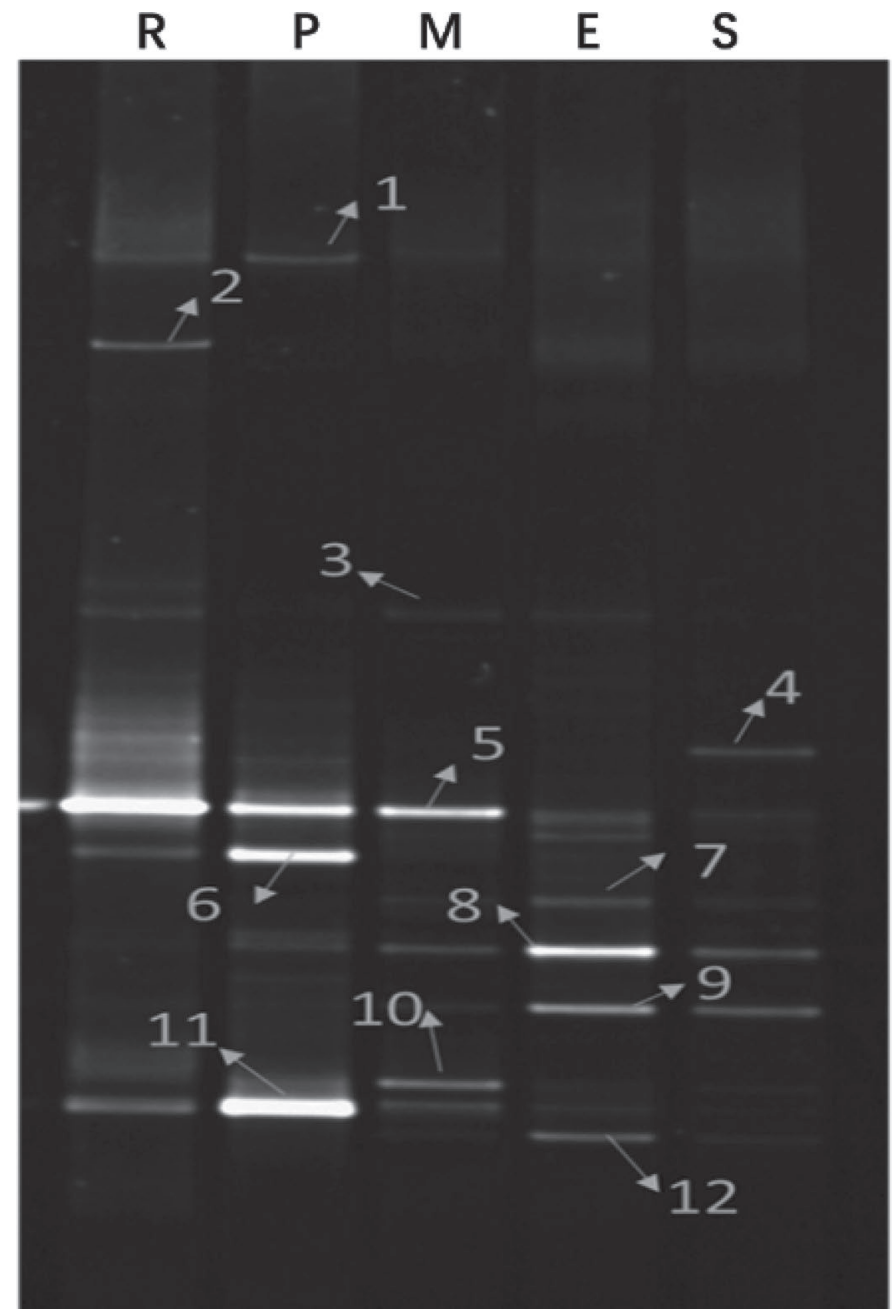

Figure 1. Denaturing gel gradient electrophoresis pattern of the V3-V5 hypervariable region of bacterial 16S rRNA gene from different stages of powdered infant formula production. Bands with a number were sequenced and identified. $\mathrm{R}=$ raw milk; $\mathrm{P}=$ pasteurization; $\mathrm{M}$ $=$ mixing; $\mathrm{E}=$ evaporation; $\mathrm{S}=$ spray-drying.
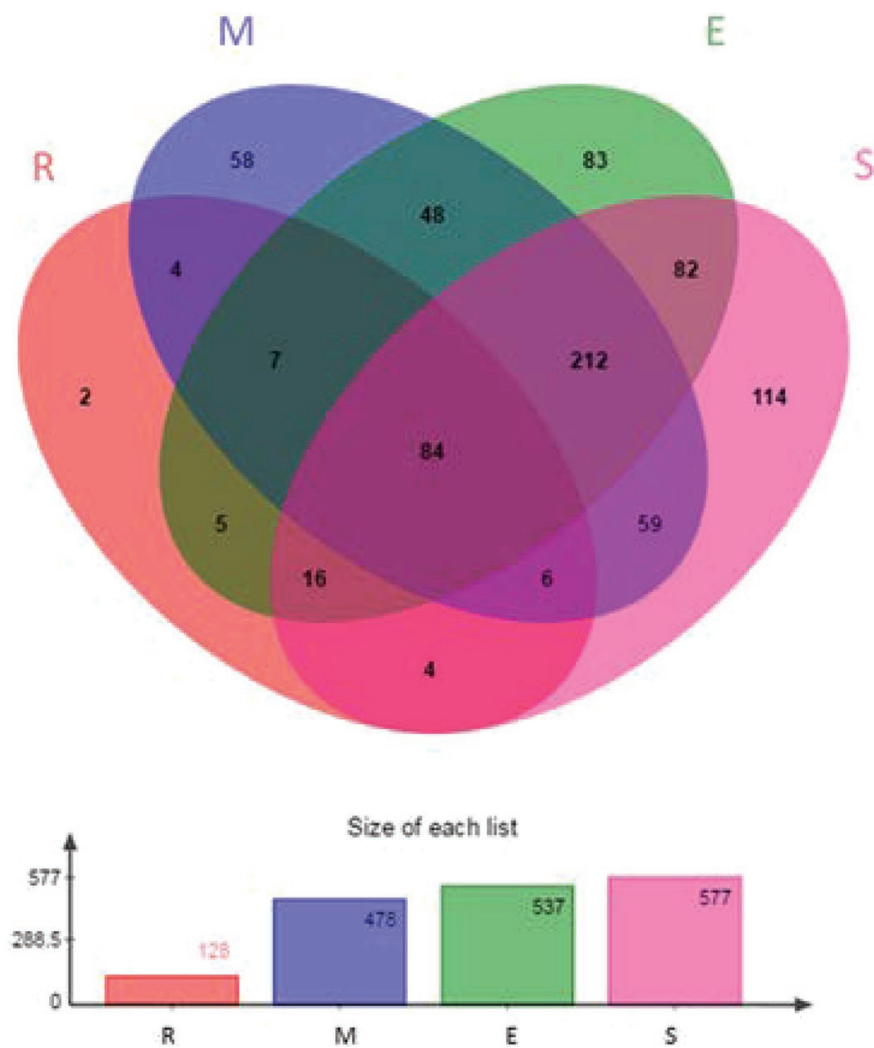

Figure 2. Venn diagram of operational taxonomic units that are shared or unique for different processes of powdered infant formula production. $\mathrm{R}=$ raw milk; $\mathrm{M}=$ mixing; $\mathrm{E}=$ evaporation; $\mathrm{S}=$ spraydrying.

and spray-drying stages, the 3 indices were almost similar, suggesting close bacterial richness and diversity. The number of OTU increased from the raw milk stage to the spray-drying stage, suggesting a potential risk of microbial contamination during PIF production.

The composition of bacterial communities was different at the 4 stages (Supplemental Figure S5; https://doi .org/10.3168/jds.2019-18064). According to amplicon analysis, Proteobacteria was the highest abundant phylum $(99.07 \%)$ at the raw milk stage and was present at all stages of PIF production (Supplemental Figure S5). Firmicutes was also an abundant phylum in the mixing stage $(76.51 \%)$, the evaporation stage $(69.66 \%)$, and the spray-drying stage $(64.83 \%)$. The cell walls of Firmicutes are thick and have high peptidoglycan content. Therefore, heat-resistant Lactobacillus and Streptococcus can resist pasteurization and survive to affect the quality of the milk powder (Christiansen et al., 2006; Stephanie and Dennis, 2010). Deinococcus-Thermus and Bacteroidetes also showed high abundance, similar to the findings of Zhang et al. (2015), who reported that Actinobacteria, Firmicutes, Proteobacteria, and Bacteroidetes were dominant in the microbial populations of 
raw milk from dairy cows. Pseudomonas was the most abundant genus at the raw milk stage (96.79\%). Lactococcus $(42.22 \%)$ and Streptococcus $(21.45 \%)$ showed high abundance at the mixing, evaporation, and spray- drying stages (Supplemental Figure S5). Other studies have shown that Pseudomonas are linked to the formation of biofilm and spoilage (Scatamburlo et al., 2015). The cold storage of milk before heat treatment
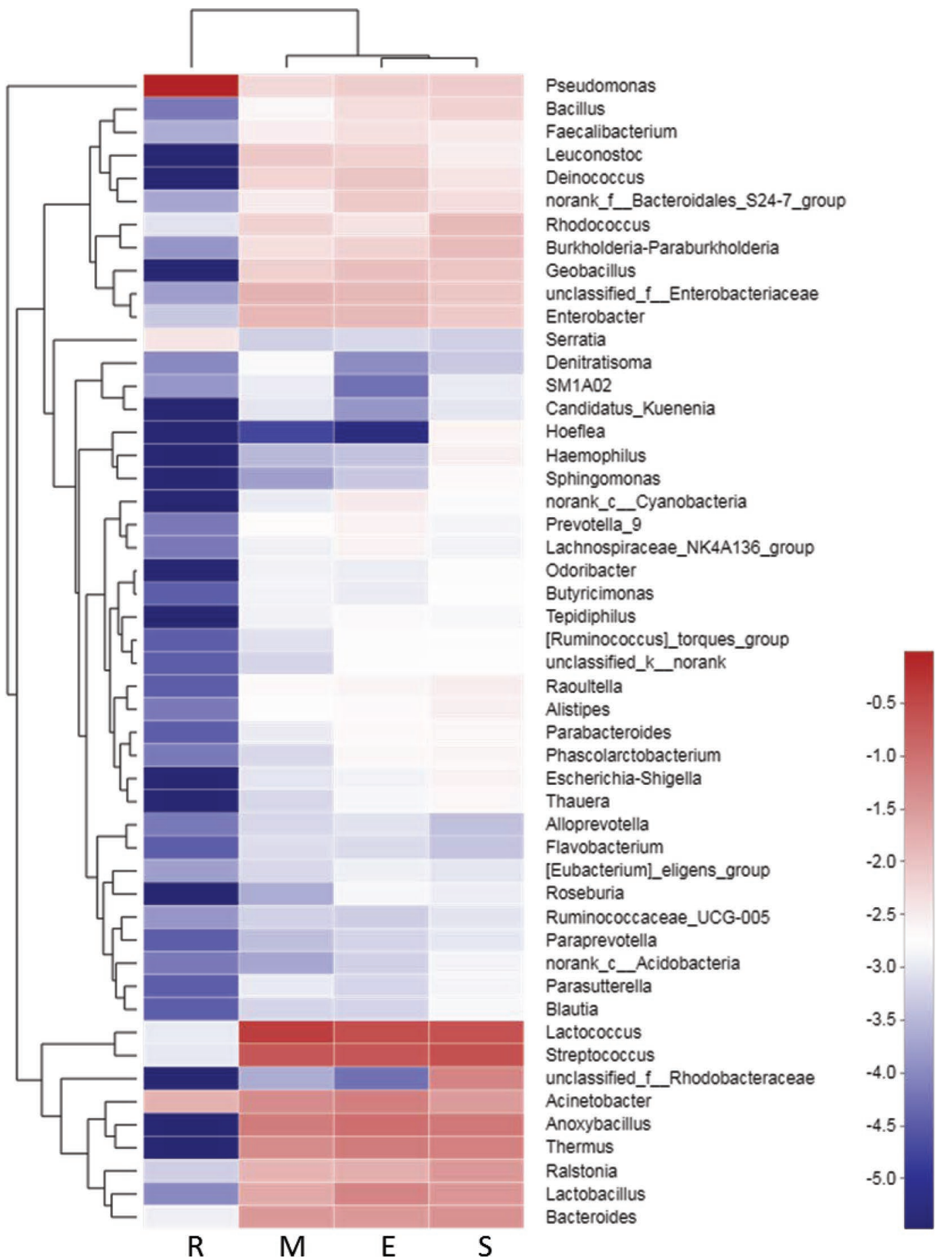

Figure 3. Relative bacterial abundance in powdered infant formula at the genus level using a heatmap. Top 50 species in terms of total abundance. The dendrogram indicates hierarchical clustering between bacterial species and powdered infant formula samples. $\mathrm{R}=$ raw milk; $\mathrm{M}$ = mixing; $\mathrm{E}=$ evaporation; $\mathrm{S}=$ spray-drying. 
in the dairy chain could lead to selective enrichment of psychrotolerant bacteria (mainly Pseudomonas). These bacteria can secrete thermoresistant proteases and lipases, resulting in spoilage and structural defects in various dairy products (Van Tassell et al., 2012; Stoeckel et al., 2016).

The heatmap of Figure 3 depicts hierarchical clustering between bacterial species and between PIF samples with similar species proportions, suggesting the close clustering of production stages. From sample clustering, we divided the 4 stages into 2 large branches, and found that the raw milk stage was only stage that differed from the others (Figure 3). Pseudomonas was the main genus in the raw milk stage: the grid for that genus had the deepest color. In the other 3 stages, Lactococcus, Streptococcus, Acinetobacter, Anoxybacillus, Thermus, and Lactobacillus showed similar colors (pink). Interestingly, Acinetobacter had a good degree of color correlation across the 4 stages. Compared with the raw milk stage, the other 3 stages had close distance in flora composition. The top 50 species in terms of total abundance (Figure 3) were similar across the 3 stages, but the others showed significant differences (Supplemental Figure S5; https://doi.org/10.3168/jds .2019-18064). Comparing the mixing and evaporation stages (Figure 3 and Supplemental Figure S5), we found no differences in Streptococcus, Bacteroides, or Enterobacter abundance, but distinct differences for Unclassified_f_Enterobacteriaceae. Ralstonia showed an obvious difference $(P<0.01)$, and the others had highly significant differences $(P<0.001)$. Comparing the evaporation and spray-drying stages (Figure 3 and Supplemental Figure S5), we found no difference in abundance for Pseudomonas; Geobacillus was distinct in the 2 stages $(P<0.05)$, and the other species showed highly significant differences.

High-throughput sequencing is a very powerful tool for examining microbial population structure and dynamics in diverse ecosystems. Bacterial diversity by HTS was significantly greater than by PCR-DGGE in the present study. The differences in bacterial communities during PIF production as shown by DGGE and HTS can be attributed to the brevity of DGGE data and the different hypervariable regions of $16 \mathrm{~S}$ rRNA selected by these 2 methods. A species whose abundance is less than $1 \%$ of the total microbial abundance usually cannot be accurately discerned using DGGE (Logares et al., 2014), so it will miss a large number of rare species with low abundance (Hill and Smythe, 2012). Higher amplification specificity and more accurate identification by database search could help to discern bacterial communities in various ecosystems (Stackebrandt, 2006), but not all DGGE bands will be retrieved because some are difficult to recognize. Thus,
DGGE usually generates much less data than HTS (Foucher et al., 2004). In addition, the same horizontal bands in a DGGE gel may not indicate the same species (Hong et al., 2007). However, we found no such phenomenon in the present study. Based on DGGE and HTS, we identified Pseudomonas fluorescens as the dominant microorganism at the raw milk stage. Apart from some Enterococcus and Streptococcus spp., most bacteria (e.g., mesophilic Lactobacillus spp.) were killed by pasteurization. After pasteurization, the proportion of B. licheniformis significantly increased and Bacillus became the main genus, followed by Pseudomonas, Lactococcus, and Streptococcus. The mixing, evaporation, and spray-drying stages had similar bacterial richness but were less similar in terms of species: the main species were Enterococcus faecium, L. lactis, Lactobacillus plantarum, and B. licheniformis. We found no indicative pathogenic bacteria during PIF production, similar to the report by Hou et al. (2015). Our study is the first to offer insights into the dynamic changes in microbial diversity during PIF production.

\section{ACKNOWLEDGMENTS}

This work was supported by the National Key R\&D Program of China (grant No. 2018YFC1604305 and 2018YFD0502306), National Natural Science Foundation of China (grant No. 31871776, 31972101 and 31771956), Natural Science Foundation of Shanghai (grant No. 18ZR1426800), Shanghai Agriculture Applied Technology Development Program (Grant No. 2019-02-08-00-07-F01152), and Shanghai Engineering Research Center of Food Microbiology (grant No. 19DZ2281100). The authors have not stated any conflicts of interest.

\section{REFERENCES}

Aponte, M., V. Fusco, R. Andolfi, and S. Coppola. 2008. Lactic acid bacteria occurring during manufacture and ripening of Provolone del Monaco cheese: Detection by different analytical approaches. Int. Dairy J. 18:403-413. https://doi.org/10.1016/j.idairyj.2007.10 .011 .

Callon, C., F. Duthoit, C. Delbès, M. Ferrand, Y. L. Le Frileux, R. D. De Crémoux, and M. C. Montel. 2007. Stability of microbial communities in goat milk during a lactation year: Molecular approaches. Syst. Appl. Microbiol. 30:547-560. https://doi.org/10 .1016/j.syapm.2007.05.004.

Christiansen, P., E. Waagner Nielsen, F. K. Vogensen, C. H. Brogren, and Y. Ardö. 2006. Heat resistance of Lactobacillus paracasei isolated from semi-hard cheese made of pasteurised milk. Int. Dairy J. 16:1196-1204. https://doi.org/10.1016/j.idairyj.2005.10.009.

Delgado, S., C. T. Rachid, E. Fernández, T. Rychlik, A. Alegría, R. S. Peixoto, and B. Mayo. 2013. Diversity of thermophilic bacteria in raw, pasteurized and selectively-cultured milk, as assessed by culturing, PCR-DGGE and pyrosequencing. Food Microbiol. 36:103-111. https://doi.org/10.1016/j.fm.2013.04.015.

Doyle, C. J., D. Gleeson, P. W. O'Toole, and P. D. Cotter. 2016. High-throughput sequencing highlights the significant influence of 
seasonal housing and teat preparation on the raw milk microbiota. Appl. Environ. Microbiol. 83:e02694-16. https://doi.org/10.1128/ AEM.02694-16.

Foucher, A. L. J. L., T. Bongers, L. R. Noble, and M. J. Wilson. 2004 Assessment of nematode biodiversity using DGGE of $18 \mathrm{~S}$ rDNA following extraction of nematodes from soil. Soil Biol. Biochem. 36:2027-2032. https://doi.org/10.1016/j.soilbio.2004.05.021.

Hantsis-Zacharov, E., and M. Halpern. 2007. Culturable psychrotrophic bacterial communities in raw milk and their proteolytic and lipolytic traits. Appl. Environ. Microbiol. 73:7162-7168. https:// doi.org/10.1128/AEM.00866-07.

Hill, B. M., and B. W. Smythe. 2012. Endospores of thermophilic bacteria in ingredient milk powders and their significance to the manufacture of sterilized milk products: An industrial perspective. Food Rev. Int. 28:299-312. https://doi.org/10.1080/87559129.2011 .635487 .

Hong, H., A. Pruden, and K. F. Reardon. 2007. Comparison of CESSCP and DGGE for monitoring a complex microbial community remediating mine drainage. J. Microbiol. Methods 69:52-64. https: //doi.org/10.1016/j.mimet.2006.11.016.

Hou, Q., H. Xu, Y. Zheng, X. Xi, L. Y. Kwok, Z. Sun, H. Zhang, and W. Zhang. 2015. Evaluation of bacterial contamination in raw milk, ultra-high temperature milk and infant formula using single molecule, real-time sequencing technology. J. Dairy Sci. 98:84648472. https://doi.org/10.3168/jds.2015-9886.

Logares, R., S. Audic, D. Bass, L. Bittner, C. Boutte, R. Christen, J. M. Claverie, J. Decelle, J. R. Dolan, M. Dunthorn, B. Edvardsen, A. Gobet, W. H. C. F. Kooistra, F. Mahé, F. Not, H. Ogata, J. Pawlowski, M. C. Pernice, S. Romac, K. Shalchian-Tabrizi, N. Simon, T. Stoeck, S. Santini, R. Siano, P. Wincker, A. Zingone, T. A. Richards, C. de Vargas, and R. Massana. 2014. Patterns of rare and abundant marine microbial eukaryotes. Curr. Biol. 24:813-821. https://doi.org/10.1016/j.cub.2014.02.050.

Quigley, L., O. O'Sullivan, T. P. Beresford, R. P. Ross, G. F. Fitzgerald, and P. D. Cotter. 2012. High-throughput sequencing for detection of subpopulations of bacteria not previously associated with artisanal cheeses. Appl. Environ. Microbiol. 78:5717-5723. https:/ /doi.org/10.1128/AEM.00918-12.

Sadiq, F. A., S. Flint, L. Yuan, Y. Li, T. Liu, and G. He. 2017. Propensity for biofilm formation by aerobic mesophilic and thermophilic spore forming bacteria isolated from Chinese milk powders. Int. J. Food Microbiol. 262:89-98. https://doi.org/10.1016/j.ijfoodmicro 2017.09.015

Scatamburlo, T. M., A. K. Yamazi, V. Q. Cavicchioli, F. A. Pieri, and L. A. Nero. 2015. Spoilage potential of Pseudomonas species isolated from goat milk. J. Dairy Sci. 98:759-764. https://doi.org/ $10.3168 / j d s .2014-8747$.
Stackebrandt, E. 2006. Taxonomic parameters revisited: Tarnished gold standards. Microbiol. Today Nov 6:152-155.

Stephanie, D., and W. Dennis. 2010. Heat resistance of Sporolactobacillus inulinus. J. Food Sci. 46:810-812.

Stoeckel, M., M. Lidolt, V. Achberger, C. Glück, M. Krewinkel, T. Stressler, M. von Neubeck, M. Wenning, S. Scherer, L. Fischer, and J. Hinrichs. 2016. Growth of Pseudomonas weihenstephanensis, Pseudomonas proteolytica and Pseudomonas sp. in raw milk: Impact of residual heat-stable enzyme activity on stability of UHT milk during shelf-life. Int. Dairy J. 59:20-28. https://doi.org/10 .1016/j.idairyj.2016.02.045.

Van Tassell, J. A., N. H. Martin, S. C. Murphy, M. Wiedmann, K. J. Boor, and R. A. Ivy. 2012. Evaluation of various selective media for the detection of Pseudomonas species in pasteurized milk. J. Dairy Sci. 95:1568-1574. https://doi.org/10.3168/jds.2011-4958.

Wu, S., Y. Jiang, B. Lou, J. Feng, Y. Zhou, L. Guo, S. J. Forsythe, and C. Man. 2018. Microbial community structure and distribution in the air of a powdered infant formula factory based on cultivation and high-throughput sequence methods. J. Dairy Sci. 101:69156926. https://doi.org/10.3168/jds.2017-13968.

Yuan, D. D., G. C. Liu, D. Y. Ren, D. Zhang, L. Zhao, C. P. Kan, Y. Z. Yang, W. Ma, Y. Li, and L. B. Zhang. 2012. A survey on occurrence of thermophilic bacilli in commercial milk powders in China. Food Control 25:752-757. https://doi.org/10.1016/j.foodcont.2011 .12 .020 .

Zhang, R., W. Huo, W. Zhu, and S. Mao. 2015. Characterization of bacterial community of raw milk from dairy cows during subacute ruminal acidosis challenge by high-throughput sequencing. J. Sci. Food Agric. 95:1072-1079. https://doi.org/10.1002/jsfa.6800.

Zheng, Y., X. Xi, H. Xu, Q. Hou, Y. Bian, Z. Yu, L. Y. Kwok, W. Zhang, Z. Sun, and H. Zhang. 2016. Using PacBio long-read highthroughput microbial gene amplicon sequencing to evaluate infant formula safety. J. Agric. Food Chem. 64:6993-7001. https://doi .org/10.1021/acs.jafc.6b01817.

Ziyaina, M., B. N. Govindan, B. Rasco, T. Coffey, and S. S. Sablani. 2018. Monitoring shelf life of pasteurized whole milk under refrigerated storage conditions: Predictive models for quality loss. J. Food Sci. 83:409-418. https://doi.org/10.1111/1750-3841.13981.

\section{ORCIDS}

Zhi-Qiang Xiong @ (ttps://orcid.org/0000-0002-7984-3662

Yong-Jun Xia @ https://orcid.org/0000-0002-2727-2730

Lian-Zhong Ai ๑ https://orcid.org/0000-0002-6681-9102 